\title{
Semiotik und Gegenübertragung: Differenzierung der Gegenübertragung anhand der Zeichentheorie von Charles S. Peirce
}

\author{
Rosmarie Barwinski (Winterthur)
}

Zusammenfassung: Die Psychoanalyse hat sich von Beginn an mit den Begriffen Repräsentanz und Repräsentation beschäftigt (vgl. Kurthen, 2002). In den letzten Jahren sind vor allem präsymbolische Formen der Repräsentation in der theoretischen Auseinandersetzung in den Vordergrund gerückt (vgl. Beebe \& Lachmann, 1994; Stern, 1998). Es liegt nahe, für die Differenzierung unterschiedlicher Formen der Repräsentation Konzeptualisierungen aus der Semiotik heranzuziehen. Eine Theorie, die sich besonders für das Verständnis unterschiedlicher Ebenen der Repräsentation eignet, ist die Zeichentheorie von Charles S. Peirce. Wie sie dazu genutzt werden kann, um die Gegenübertragung zu differenzieren, ist Inhalt der folgenden Überlegungen. Im Artikel wird von der Hypothese ausgegangen, dass die Gegenübertragung wesentlich durch die Form der Repräsentation geprägt wird, die das Entwicklungsniveau von Ich, Selbst und Objektbeziehungen des gestörten Persönlichkeitsanteils des Patienten aufweist.

Schlüsselwörter: Gegenübertragung, präödipale Störungen, Repräsentation, Semiotik, Trauma

\section{Repräsentanz und Repräsentation präsymbolischer und symbolischer Formen der Erinnerung}

Die Begriffe Repräsentanz und Repräsentation sind früh in der psychoanalytischen Theoriebildung bedeutsam geworden. Freud selbst vermeidet weitgehend den Begriff der Repräsentation und benutzt stattdessen meist «Vorstellung». Kurthen (2002) differenziert die beiden Begriffe im «Handbuch psychoanalytischer Grundbegriffe» wie folgt:

Repräsentanz meint Repräsentieren als primär funktionales Vertreten, Repräsentation meint Repräsentieren als primär formales Wiedergegenwärtigen (Darstellen). Eine Entität kann reprä- 
sentiert werden, indem ihre Funktionen und Ziele, in einen anderen Kontext «übersetzt», von einer anderen Entität wahrgenommen werden [zum Beispiel kann der Analytiker für den Patienten in der Übertragung den Vater repräsentieren; R. B.]. Eine Entität kann aber auch repräsentiert werden, indem sie unter Erhaltung ihrer strukturellen und formalen Charakteristika in einem anderen Substrat oder Kontext abgebildet und insofern wiedergegenwärtig wird [zum Beispiel präsymbolische gegenwärtige symbolische Formen der Repräsentation; R. B.]. Ersteres wäre Repräsentanz, letzteres Repräsentation. (Kurthen, 2002, S. 616)

Wenn wir Konzepte aus der Semiotik heranziehen, beziehen wir uns auf den Begriff der Repräsentation. Wir versuchen zu verstehen, wie bestimmte psychische Erfahrungen auf unterschiedlichen Ebenen der Repräsentation vermittelt werden. In den letzten Jahren sind vor allem präsymbolische Formen der Repräsentation in der theoretischen Auseinandersetzung in den Vordergrund gerückt. Im Zusammenhang mit der Untersuchung präödipaler Störungen wurden verschiedene Konzeptualisierungen entwickelt, die den therapeutischen Umgang mit sensomotorisch kodierten Beziehungserfahrungen zu erfassen versuchen, wie z. B. "Momente der Begegnung» (Stern, 1998, S. 905) und «erhöhte affektive Momente» (Beebe \& Lachmann, 1994, S. 128). Eine wichtige Präzisierung der analytischen Theorie der Beziehung, die für die Fragestellung der vorliegenden Arbeit von Bedeutung ist, wurde von Ulrich Moser (2001) unter Zuhilfenahme neuerer Erkenntnisse aus der Affekt- und Cognitive ScienceForschung vorgenommen. Moser unterscheidet parallel ablaufende Beziehungsund Übertragungserfahrungen. Neben der Als-ob-Welt oder der «Mikrowelt» der Übertragung gibt es noch eine Beziehungsregulierung, der nichtbewusstes implizites Beziehungswissen zugrunde liegt. Unmittelbare Affektansteckung und implizite emotionale Konditionierungen in Form von Ko-Regulationen beherrschen das Beziehungsfeld. Auf diese Vorgänge reagieren beide Teilnehmer der analytischen Dyade zumeist weitgehend automatisch und häufig werden sie sich auch der dabei ablaufenden Vorgänge nicht bewusst. ${ }^{1}$ Wichtig ist, dass diese von nonverbalen, sensorischen und affektiven Aspekten von Interaktion und Kommunikation gestalteten Übertragungen innerhalb der Beziehungsregulierung nicht identisch sind mit den Vorgängen von Übertragung und Gegenübertragung in der Mikrowelt simulierter Als-ob-Prozesse. Erstere befinden sich auf einer präsymbolischen Ebene, während Letztere die Symbolisierung der Erfahrung voraussetzen. Verbale Interventionen 
scheinen in diesen Situationen mit viel zu starker Verzögerung zu erfolgen, selbst wenn die Stimme beruhigend klingt. Während bei einer Aktualisierung und Übertragung deklarativ autobiografischer Erinnerungen, die in der Kindheit zu einem späteren Zeitpunkt gebildet wurden und sprachlich abrufbar und reflektierbar sind, symbolisierende Arbeit sinnvoll und erwünscht ist, besteht die analytische Arbeit bei Patienten mit Frühstörungsanteilen vor allem darin, bislang Unsymbolisiertes erstmalig zu bildlichen und sprachlichen Symbolen werden zu lassen. Erst dann wird eine Handlungsverzögerung, ein Nachdenken-Können möglich, das mit selbstreflexiven Prozessen einhergeht.

\section{Semiotik und Repräsentation}

Um präsymbolische und symbolische Ebenen der Repräsentation zu unterscheiden, können Konzepte der Semiotik hilfreich sein. Eine Theorie, die sich besonders für das Verständnis unterschiedlicher Formen der Repräsentation eignet, ist die Zeichentheorie von Charles S. Peirce. Peirce hat eine Typologie von Zeichensystemen entwickelt, die im Wesentlichen drei Stufen umfasst: ikonisch, indexikalisch und symbolisch.

Ikonisch (bildhaft): Auf der ikonischen Ebene bildet das Zeichen das Bezeichnete ab. Es weist - wie Peirce es nennt - «Ähnlichkeiten» mit dem Objekt auf. Das Zeichen als Ikon, als Bild drückt sein Bezeichnetes unmittelbar aus. Das Bild stellt in sich das Bezeichnete dar.

Eine stärkere Ablösung des Zeichens vom Bezeichneten tritt auf der indexikalischen Ebene ein. Hier wird das Zeichen zum Anzeichen bzw. Hinweis auf das Objekt, auf das Bezeichnete. Paraverbale Kommunikation wie Mimik oder Gestik sind Beispiele für diese Ebene.

Im symbolischen Zeichensystem ist die natürliche Verbindung von Zeichen und Bezeichnetem fast vollständig aufgelöst. Das Symbol ist willkürlich im Sinne von nicht abbildlich. Die Sprache zum Beispiel entspricht dieser Repräsentationsebene. Die Zuordnung ist konventioneller Natur und bestimmt durch das jeweilige sprachliche Zeichensystem. Ob die Kuh nun vache oder cow genannt wird, steht in keiner Beziehung zum realen Objekt. Erst relativ spät in ihrer Entwicklung verstehen Kinder die Konventionalität der symbolischen Zeichen. Fragt man ein vierjähriges Kind, dem eine Kuh gezeigt wird, warum diese Kuh heisst und nicht etwa Pferd, so fällt die Antwort etwa so aus: «Das sieht doch jeder, dass dies eine Kuh ist und kein Pferd». Wie bei den Pränominalisten im europäischen Mittelalter wird der Name als natürlicher Bestandteil des Gegenstands angesehen (vgl. Fischer, 2008). 


\section{Differenzierung der Gegenübertragung anhand der Stufen}

des Zeichensystems am Beispiel des Integrationsprozesses

\section{traumatischer Erfahrungen}

Wie die Gegenübertragung entsprechend der genannten Stufen der Typologie von Zeichensystemen von Charles S. Peirce-ikonisch, indexikalisch und symbolisch - differenziert werden kann, soll im Folgenden anhand eines Beispiels illustriert werden: des Integrationsprozesses traumatischer Erfahrungen. Dieses Beispiel eignet sich besonders, da mit dem Prozess der Traumaverarbeitung sehr unterschiedliche Gegenübertragungsmanifestationen einhergehen. Wie an anderer Stelle ausgeführt (Barwinski, 2009; 2014), erlaubt die Gegenübertragung Aussagen über das Strukturniveau des Patienten und hat Indikatorfunktion für den Stand im Traumaverarbeitungsprozess. In beiden Bereichen, in der Traumabearbeitung und bei frühen Störungen, liegen intrasystemische Regulationsstörungen vor, die mit präsymbolischen Formen der Erfahrungsrepräsentation einhergehen und entweder auf Entwicklungsdefizite oder - bei der Traumaverarbeitung - auf Regressionsprozesse zurück zu führen sind.

\section{Die psychische Integration traumatischer Erfahrungen}

Die Integration traumatischer Erfahrungen kann als Symbolisierungsprozess beschrieben werden, in dem Erinnerungen an traumatische Geschehnisse über präsymbolische Ebenen der Repräsentation - in Form von Körpersymptomen und Handlungen - allmählich in Worten beschreibbar werden, ohne dass die in der Situation erlebten heftigen Gefühle ${ }^{2}$ mit der Erinnerung reaktiviert werden. Stufen, die dieser Symbolisierungsprozess durchlaufen muss, werden im TraumaIntegrationsmodell (Barwinski \& Fäh, 2005) beschrieben, das im Folgenden kurz zusammengefasst dargestellt wird.

\section{Trauma-Integrationsmodell}

Der Prozess der psychischen Integration traumatischer Erfahrungen kann in zwei Veränderungszyklen gegliedert werden, die mit unterschiedlichen psychischen Prozessen einhergehen. Im ersten Zyklus geht es um die kognitive und emotionaleWahrnehmung der traumatischen Situation, um Prozesse der Semiose, der Bild- und Sprachfindung. Im zweiten Zyklus steht die Erarbeitung der subjektiven Bedeutung traumatischer Situationen im Vordergrund sowie die Lösung von Konflikten, die mit diesen Fantasien einhergehen. 


\section{Erster Zyklus}

Unverarbeitete traumatische Erfahrungen sind im impliziten und einer frühen Form des episodischen Gedächtnisses gespeichert. Sie sind weitgehend nur sensomotorisch kodiert und können deshalb nicht erzählt werden. Die fehlende oder schwache Symbolisierung führt dazu, dass sich die Wiederholung früher Traumata im Bereich der direkten Beziehung zwischen Analytiker und Patient abspielt, zum Beispiel in Form einer Übertragungsinszenierung (Holderegger, 2012). Erstes Behandlungsziel ist dementsprechend die traumatische Erfahrung gewissermassen zu «psychisieren». Dieser Vorgang impliziert einen bedeutenden Veränderungsschritt: den Übergang von der Handlung zur Vorstellung, zur psychischen Repräsentation.

Übertragen wir die von Peirce vorgeschlagene Typologie von Zeichensystemen auf den Vorgang der Repräsentation traumatischer Erfahrungen, können für den ersten Zyklus im Trauma-Integrationsmodell folgende Aussagen gemacht werden: Wie ausgeführt, stehen im ersten Zyklus Prozesse der Semiose, der Bildund Sprachfindung, im Vordergrund. Hier geht es also nicht um die Verdrängung der Erkenntnis (wie bei der neurotischen Konfliktverarbeitung), sondern der erkennenden Sprachfindung. Das heisst, es geht um Prozesse, die die ikonische Ebene in die indexikalische, und schliesslich in die symbolische Ebene überführen.

Nach Peirce weist das Zeichen auf der ikonischen Ebene grosse Ähnlichkeiten mit dem Bezeichneten auf. Ein Beispiel für die ikonische Ebene beim Traumaintegrationsprozess sind Albträume, die weitgehende Abbildungen des traumatischen Geschehens sind (Barwinski, 2006). Bezeichnend für diese Träume ist, dass sie mit starken Affekten verknüpft sind. Wir wachen während eines Albtraums in der Regel auf, weil es im Traum nicht gelingt, die heftigen Affekte zu mildern. Diese Beobachtung legt die Hypothese nahe, dass auf der ikonischen Ebene der Repräsentation die Affekte stärker sind als auf der symbolischen Ebene. Aufgrund dieser Beobachtung kann der Schluss gezogen werden, dass mit abnehmender «Ähnlichkeit» zwischen Zeichen und Bezeichnetem auch die Affektintensität abnimmt.

Erinnerungen auf ikonischer Ebene entsprechen vermutlich dem, was Eckhardt-Henn (2002) als mit traumatischen Erlebnissen verbundenes, "psychisch unverdautes Material» bezeichnet, «welches nicht in Narrative umgesetzt und encodiert werden konnte» (S. 143). «Verdaut» werden die «Beta-Elemente» des Patienten (um einen Ausdruck von Bion [1962] zu gebrauchen, der meiner Meinung nach wesentliche Eigenschaften erfasst, die für in ikonischer Form repräsentiertes Material spezifisch sind), über die Transformation der projektiven 
Identifizierungen des Patienten (vgl. Ferro, 2003). Sie ist die Aufgabe des Analytikers. Zentral wird damit die Gegenübertragung des Analytikers, seine Empfänglichkeit oder Unzugänglichkeit für die projektiven Identifikationen des Patienten und seine Fähigkeit, die von Bion beschriebenen Beta-Elemente des Patienten durch seine psychische Aktivität zu modifizieren.

Entsprechend sind im ersten Behandlungszyklus für den Analytiker / die Analytikerin Gegenübertragungsgefühle sehr belastend. Zum Beispiel versetzt der Traumabericht des Patienten den Analytiker in ähnliche - wenn auch in ihrer Intensität abgemilderte-Gefühle, wie sie der Patient in der traumatischen Situation erlebte. Miller, Stiff und Ellis (1988) sprechen von «emotionaler Ansteckung», um diesen Vorgang zu erfassen. Die Differenzierung zwischen Selbst und Objekt in der analytischen Beziehung geht beim Analytiker bzw. der Analytikerin verloren: Der/ die AnalytikerIn identifiziert sich in einer sehr globalen Weise mit dem Patienten. Das heisst, dass mit der Ähnlichkeit des Zeichens mit dem Bezeichneten auch die Differenzierung zwischen Selbst und Objekt in der Gegenübertragung verloren zu gehen droht. Ein Beispiel, das diese Hypothese zu bestätigen scheint, ist ein typisches Gegenübertragungsphänomen des ersten Zyklus der Traumabearbeitung: Der/die AnalytikerIn erlebt stellvertretend für den Patienten dessen Gefühle. Mittels projektiver Identifikation wird der/die AnalytikerIn zum Teilobjekt des Patienten, der/die die Gefühle des Patienten in sich wahrnimmt und ihm in «verdauter» Form zurückgeben muss.

Der Übergang vom ersten zum zweiten Zyklus zeigt sich auf der indexikalischen Ebene. Über den Handlungsdialog wird die traumatische Erfahrung auf eine symbolische Ebene überführt. Ich möchte diesen Übergang mit einem Beispiel verdeutlichen:

Frau M., die schwere sexuelle Übergriffe in ihrer Kindheit erleben musste, schilderte zu Beginn ihrer Behandlung folgende Schwierigkeit: Sie hatte Angst vor den Blicken - den Augen - der Menschen. Sie hatte Angst, die Leute könnten «sehen», was sie denkt. Sie sagte: «Ich kann nicht zwischen innen und aussen unterscheiden. Ich fühle mich so, als könne man Inneres nach aussen kehren und umgekehrt.» Wie sich später zeigen sollte, spiegelte diese Angst einen Aspekt der traumatischen Situation wider (die Blicke des Täters, «mit denen er sie auszog»), aber gleichzeitig auch eine Form der Erlebnisverarbeitung: Die Angst vor ihren eigenen Gefühlen wehrte sie ab, indem sie sie als Bedrohung in der Aussenwelt wahrnahm. Zu diesem Zeitpunkt war ihr noch nicht bewusst, dass ihre heftigen Affekte in engerVerbindung mit Erlebniszuständen aus derVergangenheit standen. Bevor diese Einsicht möglich wurde, leistete sie einen wichtigen Erkenntnisschritt. 
Sie entschloss sich kurzfristig, ein paar Tage ans Meer zu fahren, um «Abstand zu bekommen». Als sie danach wieder zur Sitzung kam, berichtete sie von folgender Einsicht: «Es ging mir nicht schlecht, am ersten Tag sogar besser, aber ich habe gemerkt, dass ich nicht vor meinen Problemen weglaufen kann. Sie sind wirklich in mir - man kann Inneres nicht einfach aussen zurücklassen. Ich habe gespürt, dass da Ängste in mir sind - dass die Gefahr nicht von aussen kommt. Das erleichtert mich, weil mir dann die Menschen, vor allem die Blicke, nicht mehr so viel Angst machen, aber es bedrückt mich auch: Ich kann vor den Anderen weglaufen, aber ich kann nicht vor mir selbst davonkommen.» Sie begann zwischen innerpsychischer und äusserer Realität zu unterscheiden. Über die Überprüfung ihrer Gefühle in einer anderen Umgebung war es ihr möglich geworden, die eigenen Gefühle als eigene psychische Verfassungen zu erkennen. Aufgabe der Analytikerin war in dieser Episode, den Handlungsdialog in Worte zu fassen. Damit wurde der Übergang von der Handlung zur symbolischen Repräsentation möglich.

Dass die Unterscheidung zwischen innerer und äusserer Realität Bedingung war, um die Konfrontation mit traumatischen Erfahrungen wagen zu können, zeigte sich in den folgenden Gesprächen. In unserer nächsten Sitzung berichtete sie von Albträumen, in denen sie die Zimmertüre nicht mehr schliessen konnte. Die Abwehr gegen die Erinnerung an traumatische Erfahrungen schien brüchig geworden zu sein. Erste Erinnerungen an sexuelle Übergriffe durch den Grossvater tauchten in der Folge als Flashbacks auf. Sie berichtete, dass sie in einer Apotheke ein Einreibemittel gekauft hatte, um ihre Erkältung zu kurieren. Als sie den Geruch der Salbe roch, wurde es ihr übel, sie hatte mit Brechreiz zu kämpfen. Sie wusste plötzlich, dass ihr Grossvater unter dem Vorwand, ihren Husten behandeln zu wollen, sie am ganzen Körper berührt hatte. Die traumatische Erfahrung konnte als Erinnerungen erkannt und damit in symbolischer Form repräsentiert beziehungsweise im autobiografischen Gedächtnis gespeichert werden.

\section{Verlust der Fähigkeit zur symbolischen Repräsentation}

Die mangelnde Selbst- und Objektdifferenzierung, die mit der Reaktivierung traumatischer Erfahrungen einhergeht, scheint Resultat einer Regressionsbewegung zu sein. Repräsentanzen können nicht mehr als Repräsentanzen wahrgenommen werden. (In der Terminologie von Peirce ausgedrückt: Die Ähnlichkeit zwischen Bezeichnetem und Zeichen nimmt zu.) Entsprechend zeigen sich Abwehrmassnahmen vor allem auf der interpersonellen Ebene. Personen oder Orte, die an die traumatische Erfahrung erinnern könnten, werden vermieden. Über äusseren Abstand wird versucht, innere psychische Distanz zu wahren. Das 
vorangehende Beispiel verdeutlicht eindrücklich diese Konkretisierung psychischer Prozesse.

Bezüglich Gegenübertragung bleibt zu bemerken, dass auf der indexikalischen Ebene die Gegenübertragung als Handlungsimpuls zum Ausdruck kommt. Sie bleibt emotional belastend für den/die AnalytikerIn, kann aber wesentlich besser über die Wahrnehmung und das Verständnis eigener Handlungsimpulse aufgelöst werden. Da die Affektintensität, die mit Erinnerungen an traumatische Geschehnisse verbunden ist, im zweiten Zyklus abgenommen hat, wird die Selbst-Objekt-Differenzierung in der analytischen Beziehung mittels des Prozesses der «reflektierenden Abstraktion» (vgl. Barwinski, 2014; Kesselring, 2010) möglich. Damit wird die Gegenübertragung als solche erkannt, anstatt unverstanden im Handlungsdialog (Klüwer, 1995) agiert zu werden. Mit dem Erkennen und Verstehen der Gegenübertragung befindet sich der/die AnalytikerIn auf der symbolischen Stufe. Es wird möglich, die Inszenierungen des Patienten mittels der Gegenübertragung zu erfassen und zu deuten, um auch dem Patienten den Übergang von der Handlung zur Vorstellung zu ermöglichen.

\section{Zweiter Zyklus}

Im zweiten Zyklus ist die symbolische Repräsentation des traumatischen Geschehens weitgehend möglich geworden. Die traumatische Situation kann wahrgenommen und verbalisiert werden. Erinnerungen an traumatische Erfahrungen gehen nicht mehr mit überwältigenden Affekten einher. Über die Repräsentation der traumatischen Geschehnisse wird auch der Aufbau von Repräsentanzen möglich (vgl. Kurthen, 2002). Sie bleiben als Repräsentanzen erhalten, was im ersten Zyklus des Traumaverarbeitungsprozesses nicht möglich war. Damit kann der Patient zwischen realer Beziehung und Übertragung unterscheiden. Er kann Übertragungsdeutungen verstehen und für den Einsichtsprozess nutzbar machen. Eine Patientin, die in ihrer Kindheit schwere Verlusterlebnisse erleiden musste, berichtete mir folgenden Traum: Sie will zu mir zur Sitzung kommen. Als sie an der richtigen Adresse ankommt, ist mein Haus verschwunden. Mit diesem Traum gelingt es der Patientin, für ihre Angst ein Bild zu finden und mir in Worten mitzuteilen, was ihr in der Beziehung zu mir Angst bereitet. Ein fliessender Übergang von einer Repräsentationsebene zur anderen war möglich geworden.

Die Gegenübertragung ist im zweiten Zyklus nicht mehr mit diesen heftigen Gefühlen verbunden wie im ersten Verarbeitungszyklus traumatischer Erfahrungen. Während bei schwach integrierten traumatischen Erfahrungen die Differenzierung zwischen Selbst und Objekt in der Beziehung zum Patienten verloren geht, wer- 
den mit zunehmendem Integrationsgrad der traumatischen Erfahrungen die Gegenübertragungsgefühle des Analytikers / der Analytikerin objektaler. Die komplementäre und konkordante Gegenübertragung (Racker, 1953) kann wesentlich besser den reaktivierten Beziehungsmustern aus der Vergangenheit zugeordnet werden. Damit befindet sich, wie bereits erwähnt, der/die AnalytikerIn auf der symbolischen Stufe. Über die Deutung der Übertragung wird auch beim Patienten der Übergang von der Handlung zur Vorstellung ermöglicht.

\section{Deutung und semiotische Ebenen}

Anzumerken bleibt, dass die genannten unterschiedlichen semiotischen Ebenen nicht nur für die Behandlung von Traumafolgestörungen oder sogenannten frühen Störungen relevant sind, sondern über vor- und aussersprachliche Momente in jeden therapeutischen Dialog mit einfliessen (vgl. Fischer, 2007). Der therapeutische Dialog findet niemals allein auf der Ebene expliziter Deutungen und Bedeutungen statt, sondern umfasst über die semantische Ebene hinaus «semiotische» Komponenten ikonischer und indexikalischer Art - in der Terminologie von Charles S. Peirce: Bildhafte Zeichen, die vor allem das ansprechen, was man das Klima oder die Atmosphäre nennen kann, sowie An-Zeichen, die auf mögliches Handeln hindeuten oder dieses veranlassen.

Bei der Bearbeitung traumatischer Erfahrungen oder auch der Behandlung sogenannter früher Störungen haben die genannten semiotischen Ebenen in dem Sinne eine grössere Bedeutung als beispielsweise bei der neurotischen Konfliktbearbeitung, da die zu bearbeitenden Erfahrungen häufig nur auf der ikonischen und indexikalischen Ebene repräsentiert sind. Dies ist bei der Konfliktverarbeitung nicht der Fall. Hier wurden Geschehnisse oder verpönte Wünsche verdrängt oder desymbolisiert. Die Symbolisierung der Erfahrung ging jedoch der Verdrängung voraus. Bevor ein therapeutischer Umgang möglich wird, wie er für die Konfliktverarbeitung üblich ist, muss hingegen bei der Traumaverarbeitung die Symbolisierung der traumatischen Geschehnisse therapeutisch unterstützt werden. Erst dann werden Übertragungsdeutungen möglich, wie dies für die Konfliktverarbeitung empfohlen wird. Diesen Sachverhalt meint vermutlich Ferro, wenn er darauf hinweist, dass die Deutung auf derselben Kommunikationsebene erfolgen muss, die der Patient benutzt. «Dies gewährleistet, dass all die semantischen Übergänge, die der Patient suggeriert, tatsächlich aufgenommen werden. Diese Kommunikationsebene ergibt sich aus dem Bewusstsein, dass die Alphabetisierung der Beta-Elemente des Patienten die Vorbedingung für jede Symbolisierung darstellt» (2003, S. 242). 
Zusammenfassend kann festgehalten werden, dass je stärker der gestörte Persönlichkeitsbereich des Patienten Repräsentationsformen aufweist, die der ikonischen und indexikalischen Stufe zugeordnet werden müssen, desto deutlicher tritt die Notwendigkeit hervor, den Handlungsaspekt der Deutung zu berücksichtigen. Deutungen werden jetzt verstärkt unter dem performatorischen Aspekt einer Sprachhandlung betrachtet bis hin zu Deutungen, die überwiegend im Medium des Handelns gegeben werden. Anders gesagt, sind explizit verbale Deutungen, die überwiegend auf den «Darstellungsaspekt» der Sprache abheben, in dem Masse kontraindiziert (weil bestenfalls unwirksam) wie das Entwicklungsniveau von Ich, Selbst und Objektbeziehungen des Patienten einen Schwerpunkt auf Repräsentationsformen der ikonischen oder indexikalischen Stufe aufweist. «Das letztlich aufklärerische Ziel des psychoanalytischen Dialogs mit dem Schwerpunkt der Deutung, Unbewusstes bewusst zu machen, wird dabei keineswegs aufgegeben, sondern um das kommunikative Medium der Handlung bzw. Inter-Aktion erweitert» (Fischer, 2008, S. 12).

\section{Gegenübertragungsmanifestationen der ikonischen, indexikalischen und symbolischen Stufe}

Abschliessend bleibt bezüglich der eingangs gestellten Fragestellung, wie die Zeichentheorie von Charles S. Peirce dazu genutzt werden kann, um die Gegenübertragung zu differenzieren, festzuhalten, dass die Gegenübertragung mit den unterschiedlichen Stufen der Repräsentation - ikonisch, indexikalisch und symbolisch - sehr unterschiedliche Eigenschaften aufweist.

Auf der ikonischen Ebene der Repräsentation ist die Gegenübertragung für den Analytiker bzw. die Analytikerin sehr belastend. Die Differenzierung zwischen Selbst und Objekt in der Beziehung zum Patienten geht beim Analytiker/bei der Analytikerin verloren. Mittels projektiver Identifikation wird der/die AnalytikerIn zum Teilobjekt des Patienten, das die Gefühle des Patienten in abgemilderter Form in sich wahrnimmt.

Auf der indexikalischen Ebene, die ich hier als Handlungsdialog beschrieben habe, zeigt sich die Gegenübertragung vor allem als Handlungsimpuls, die in der Übertragung angebotenen Rollenerwartungen zu agieren. Die Gegenübertragung zeichnet sich nicht mehr durch diese starken Affekte aus, wie sie für die ikonische Ebene typisch zu sein scheinen, und die Differenzierung zwischen Selbst und Objekt in der analytischen Beziehung ist nicht ganz verloren gegangen, droht aber im Handlungsdialog (Klüwer, 1995) agiert zu werden. 
Erst wenn der Analytiker/die Analytikerin Inszenierungen des Patienten mittels seiner Gegenübertragung als solche erkennt und versteht, ist die symbolische Ebene der Repräsentation erreicht. Auf der symbolischen Ebene bleiben psychische Repräsentanzen erhalten, das heisst, die Gegenübertragung kann vom Analytiker benannt und als Zugang zum szenischen Verstehen in der analytischen Beziehung genutzt werden.

\section{Literatur}

Barwinski, R. \& Fäh, R. (2005). Traumabearbeitung in psychoanalytischen Langzeitbehandlungen. Einzelfallstudien und Fallvergleich auf der Grundlage psychotraumatologischer Konzepte und Modelle. Kröning: Asanger.

Barwinski, R. (2014). Differenzierung der Gegenübertragung anhand entwicklungspsychologischer Konzepte. Psyche, 6, 517-536.

Barwinski, R. (2009). Die erinnerte Wirklichkeit. Zur Bedeutung von Erinnerungen im Prozess der Traumaverarbeitung. Kröning: Asanger.

Barwinski, R. (2006). Die Funktion des Traumes im Schlaf. Psychoanalytische und neurobiologische Befunde. Forum der Psychoanalyse, 1, 15-24.

Beebe, B. \& Lachmann, F. F. (1994). Representation and internalisation in infancy: The principles of salience. Psychoanalytic Psychology, 11, 127-165.

Bion, W. R. (1962). Lernen durch Erfahrung. Frankfurt a. M.: Suhrkamp.

Eckhardt-Henn, A. (2002). Dissoziation. Mertens, W. \& Waldvogel, B. (Hrsg.), Handbuch psychoanalytischer Grundbegriffe (S. 141-145). Stuttgart: Kohlhammer.

Ferro, A. (2003). Das bipersonale Feld. Giessen: Psychosozial-Verlag.

Fischer, G. (2008). Logik der Psychotherapie - philosophische Grundlagen der Psychotherapiewissenschaft. Kröning: Asanger.

Fischer, G. (2007). Kausale Psychotherapie. Kröning: Asanger.

Holderegger, H. (2012). Trauma und Übertagung. Psyche, 66, 1102-1116.

Kesselring, Th. (2010). Zur Entwicklung der Emotionen: Eine Erweiterung der Piagetschen Theorie. Zeitschrift für Psychotraumatologie, Psychotherapiewissenschaft und Psychologische Medizin, 2, 85-96.

Klüwer, R. (1995). Studien zur Fokaltherapie. Frankfurt a. M.: Suhrkamp.

Kurthen, M. (2002). Repräsentanz. Mertens, W. \&Waldvogel, B. (Hrsg.), Handbuch psychoanalytischer Grundbegriffe (S. 615-618). Stuttgart: Kohlhammer.

Miller, K., Stiff, J.B. \& Ellis, B.H. (1988). Communication and empathy as precursors to burnout among human service workers. Communication Monographs, 55, 268-283. 
Moser, U. (2001). Übertragung, Gegenübertragung und therapeutische Situation am Beispiel «früher Störungen». Psyche, 55, 97-136.

Racker, H. (1953). Contribution to the problem of countertransference. International Journal of Psychoanalysis, 38, 124-149.

Stern, D. (1998). Non interpretative mechanisms in psychoanalytic therapy. The «something more» than interpretation. International Journal of Psychoanalysis, 79, 903-921.

\section{Anmerkungen}

1 Mosers Betrachtungsweise steht in Übereinstimmung mit neueren Befunden der Gedächtnispsychologie und der Hirnforschung: Implizites Beziehungswissen wird bereits früh erworben, gilt als nicht-bewusst (im Unterschied zu psychodynamisch unbewussten Vorgängen des deklarativen autobiografischen Gedächtnisses), aber beeinflusst dennoch das bewusste Erleben, Kognizieren und Handeln. Das nicht-deklarative, implizite Gedächtnis bleibt von Säuglingstagen an (in Fragmenten schon seit fötaler Existenz) in Verhaltens- und Erlebensmuster aufbewahrt, die sich im späteren Leben als Übertragungsmuster mit implizit prozeduralen Erlebens- und Gedächtnisanteilen manifestieren. Im Handlungsdialog wird die Aktualisierung des impliziten/nichtdeklarativen Beziehungswissens seitens des Patienten vom Therapeuten dahingehend erlebt, dass er unmittelbar präsent, mit seinen Gefühlen direkt erlebbar, haltgebend, resonant sein soll.

2 Die Begriffe «Emotion» und «Gefühl» gebrauche ich synonym, obwohl manche Autoren den Begriff «Emotion» auf beobachtbares Verhalten beziehen, während der Begriff «Gefühl» für die subjektive Erfahrung verwendet wird. Der Begriff «Affekt» steht für besonders starke Emotionen (vgl. Kesselring, 2010). 